\title{
Parameterized Complexity of the Arc-Preserving Subsequence Problem*
}

\author{
Dániel Marx ${ }^{1}$ and Ildikó Schlotter ${ }^{2}$ \\ 1 Tel Aviv University, Israel \\ 2 Budapest University of Technology and Economics, Hungary \\ $\{$ dmarx, ildi\}@cs.bme.hu
}

\begin{abstract}
We study the Arc-Preserving Subsequence (APS) problem with unlimited annotations. Given two arc-annotated sequences $P$ and $T$, this problem asks if it is possible to delete characters from $T$ to obtain $P$. Since even the unary version of APS is NP-hard, we used the framework of parameterized complexity, focusing on a parameterization of this problem where the parameter is the number of deletions we can make. We present a linear-time FPT algorithm for a generalization of APS, applying techniques originally designed to give an FPT algorithm for INDUCED SubGraph IsOMORPHISM on interval graphs [12].
\end{abstract}

\section{Introduction}

Many important problems in computational biology are related to pattern matching in strings, since DNA, RNA, or protein molecules can be viewed as sequences of nucleotides or amino acids. To gain information about such molecules, we often need to compare two sequences and measure their similarity.

Given two sequences $S_{1}$ and $S_{2}$ over some alphabet, the task of the LONGEST Common Subsequence (LCS) problem is to find the longest possible sequence that is the subsequence of both $S_{1}$ and $S_{2}$. In other words, we are looking for a sequence $C$ that can be obtained both from $S_{1}$ and from $S_{2}$ by deleting characters. This problem arises in many applications, like deciding if two species are biologically related, or whether two proteins are likely to exhibit similar functionalities related to three-dimensional structure (protein folding). Another classical problem, SUBSEQUENCE, asks if a sequence is the subsequence of another.

If we only want to deal with character sequences, LCS can be solved efficiently using dynamic programming. However, recent biological research suggests that we might loose relevant information if we model DNA, RNA, or protein molecules simply as sequences. The reason for this is that in such molecules, the shape and hence the functionality is greatly affected by chemical bonds between elements that might be far apart from each other in the sequence. Arc-annotated sequences are widely used to represent such bonds. In this model, any two elements (or bases) of a sequence can be connected to each other through an arc.

\footnotetext{
* Supported by ERC Advanced Grant DMMCA and by the Hungarian National Research Fund OTKA 67651.
} 
For two arc-annotated sequences $S_{1}$ and $S_{2}$, the Longest ArC-PrEserving Common SubSEQUence or LAPCS asks for an arc-annotated sequence $C$ of maximum length that can be obtained both from $S_{1}$ and from $S_{2}$ by deleting bases together with all arcs incident to them. Since LAPCS is NP-complete even if the arc structures are highly restricted $[5,6,10]$, researchers focused on polynomial-time solvable cases and approximation algorithms [5, 10, 9, 11].

Another direction of research is to use the parameterized complexity framework $[4,7]$. This area deals with NP-hard problems by giving algorithms that have an acceptable running time on many relevant instances. An algorithm is fixed-parameter tractable (FPT) if its running time is bounded by $f(k) n^{O(1)}$ for some function $f$, where $n$ is the input size and $k$ is the parameter associated with the input. The idea behind this definition is that the running time of an FPT algorithm remains tractable provided that the parameter has small value.

Parameterized complexity of LAPCS has already been studied, and FPT algorithms were presented for various parameterizations [1,6]. An interesting parameterization is where the parameter is the number of deletions we are allowed to make in order to construct the common subsequence. This models a situation where we compare two sequences which are similar. An FPT algorithm was given in [1] with this parameter, but it only applies for a restricted case.

Unlike most previous results, we considered unlimited annotations where any two bases of a sequence can be connected by arcs. Instead of concentrating on LAPCS, we dealt with the more simple ArC-PrESERving SubSEQuence problem (APS), the annotated analog of SubSEQUENCE. Given two arc-annotated sequences $P$ and $T$, the task of APS is to find out whether the pattern sequence $P$ can be obtained by deleting some bases of the target sequence $T$, together with all the arcs incident to them. We remark that APS on its own is an interesting problem in computation biology, and has been widely studied in the literature. Its NP-hardness has been proved for numerous restricted cases [2], and polynomial-time algorithms have been presented $[8,3]$ for limited arc structures.

Here, we present an FPT algorithm for the unlimited APS, where the parameter is the number $k$ of deletions allowed. Our algorithm runs in $f(k) n$ time for some function $f$ depending only on $k$, where $n$ is the input size. In fact, we solve a generalization of APS where a few arcs can be deleted additionally. We mention that APS is W[1]-hard if the parameter is the length of the pattern [5].

The ideas and techniques applied here originate from an FPT algorithm solving a seemingly unrelated problem on interval graphs [12]. This algorithm answers the INDUCED SUBGRAPH ISOMORPHISM in FPT time: given two interval graphs $G$ and $H$ and a parameter $k$, is it possible to delete $k$ vertices from $G$ to obtains a graph isomorphic to $H$ ? Our work shows that research connected to interval graphs can be useful for arc-annotated sequences as well.

\section{Problem definition and notation}

We denote $\{1, \ldots, n\}$ by $[n]$. We refer to the elements of a sequence $S$ over an alphabet $\Sigma$ as bases. The $i$-th base of $S$ is $S[i]$, and the length of $S$ is $|S|$. 
Let $S_{P}$ and $S_{T}$ be two sequences over $\Sigma$. Let $\left|S_{P}\right|=n_{P}$ and $\left|S_{T}\right|=n_{T}$, assume $n_{P} \leq n_{T}$. We say that $S_{P}$ is a subsequence of $S_{T}$ if $S_{P}$ can be obtained by deleting bases from $S_{T}$, or equivalently, if there is a bijective mapping $\varphi$ from $\left[n_{P}\right]$ into a subset of $\left[n_{T}\right]$ such that $\varphi\left(i_{1}\right)<\varphi\left(i_{2}\right)$ for each $1 \leq i_{1}<i_{2} \leq n_{P}$, and $S_{P}[i]=S_{T}[\varphi(i)]$ for each $i \in\left[n_{P}\right]$. We call such a $\varphi$ an alignment of $\left(S_{P} ; S_{T}\right)$. We write $S^{\mathrm{del}}(\varphi)$ to denote the set of bases that have to be deleted from $S_{T}$ according to $\varphi$, i.e. $S^{\operatorname{del}}(\varphi)=\left[n_{T}\right] \backslash \bigcup_{i \in\left[n_{P}\right]} \varphi(i)$.

An arc-annotation $A$ of a sequence $S$ of length $n$ is a multiset of pairs of integers from $[n]$, where each pair $\left(i_{1}, i_{2}\right) \in A$ satisfies $i_{1}<i_{2}$. An arc-annotated sequence $(S, A)$ is a sequence $S$ together with an arc-annotation $A$ for $S$. We say that an $\operatorname{arc}\left(i_{1}, i_{2}\right)$ starts at $i_{1}$, ends at $i_{2}$, and connects the positions $i_{1}$ and $i_{2}$ incident to it. We write $A\left(i_{1}, i_{2}\right)$ for the multiplicity of the pair $\left(i_{1}, i_{2}\right)$ in $A$, and we write $A^{+}(i)$ and $A^{-}(i)$ for the set of arcs starting or ending at $i$, respectively. Also, we let $a^{\text {start }}$ and $a^{\text {end }}$ to denote the starting and ending position of an arc a. We use $|(S, A)|$ to denote the size of $(S, A)$ in binary encoding.

Given two arc-annotated sequences $\left(S_{P}, A_{P}\right)$ and $\left(S_{T}, A_{T}\right)$, we say that $\left(S_{P}, A_{P}\right)$ is an arc-preserving subsequence of $\left(S_{T}, A_{T}\right)$ if it can be obtained from $\left(S_{T}, A_{T}\right)$ by deleting bases from it, i.e. there is an alignment $\varphi$ of $\left(S_{P} ; S_{T}\right)$ such that $A_{P}(i, j)=A_{T}(\varphi(i), \varphi(j))$ for any $1 \leq i<j \leq\left|S_{P}\right|$. Such an alignment is an arc-preserving alignment of $\left(S_{P}, A_{P} ; S_{T}, A_{T}\right)$. Note that by deleting a base, we also mean the deletion of the arcs incident to it. Given two arc-annotated sequence $P$ and $T$, the Arc-Preserving Subsequence problem (APS) asks whether $P$ is an arc-preserving subsequence of $T$.

We will deal with the following generalization of APS, which we call ALmosT APS or AAPS: given two arc-annotated sequences $\left(S_{P}, A_{P}\right)$ and $\left(S_{T}, A_{T}\right)$ and some $k_{a} \in \mathbb{Z}$, we ask if we can delete some bases from $S_{T}$ (together with their incident arcs) and at most $k_{a}$ arcs in addition to obtain $\left(S_{P}, A_{P}\right)$. Formally, we have to decide if there is a set $A^{\text {del }}$ of at most $k_{a}$ arcs in $A_{T}$ such that $\left(S_{P}, A_{P}\right)$ is an arc-preserving subsequence of $\left(S_{T}, A_{T} \backslash A^{\mathrm{del}}\right)$. We call $\varphi$ a $k_{a}$-alignment for $\left(S_{P}, A_{P} ; S_{T}, A_{T}\right)$ if $\varphi$ is an arc-preserving alignment of $\left(S_{P}, A_{P} ; S_{T}, A_{T} \backslash A^{*}\right)$ for some set $A^{*}$ with $\left|A^{*}\right| \leq k_{a}$. Also, we let $A^{\text {del }}(\varphi)$ to denote such an $A^{*}$.

Given a sequence $S$, let $S^{\text {rev }}$ denote the reverse of $S$. For a position $i$ of $S$, we will use $i^{\text {rev }}$ to denote the position $|S|-i+1$ of $S^{\text {rev }}$ corresponding to $i$. If $A$ is an arc-annotation of $S$, then let $A^{\text {rev }}$ denote the corresponding arc-annotation of $S^{\text {rev }}$, meaning $A^{\text {rev }}\left(i_{1}, i_{2}\right)=A\left(i_{2}^{\text {rev }}, i_{1}^{\text {rev }}\right)$. We also let $X^{\text {rev }}=\left\{i^{\text {rev }} \mid i \in X\right\}$ for any set $X$ of positions in $S$.

If $\varphi$ is a $k_{a}$-alignment for $\left(S_{P}, A_{P} ; S_{T}, A_{T}\right)$, then $\varphi^{\text {rev }}$ is the corresponding $k_{a}$-alignment for $\left(S_{P}^{\mathrm{rev}}, A_{P}^{\mathrm{rev}} ; S_{T}^{\mathrm{rev}}, A_{T}^{\mathrm{rev}}\right)$, i.e. $\varphi^{\mathrm{rev}}(i)=\left(\varphi\left(i^{\mathrm{rev}}\right)\right)^{\text {rev }}$ for each $i$.

Due to lack of space, we omit several proofs, see the full paper for them.

\section{Fixed-parameter tractability of APS}

In this section we present an FPT algorithm for AAPS, a generalization of APS, with the parameterization where the parameters are the number of bases to delete and the number of arcs that can be deleted additionally. 
Almost Arc-Preserving Subsequence

Input: Two arc-annotated sequences $\left(S_{P}, A_{P}\right)$ and $\left(S_{T}, A_{T}\right)$, and $k_{a} \in \mathbb{Z}$.

Parameters: $k_{a}$ and $k_{b}=\left|S_{T}\right|-\left|S_{P}\right|$.

Task: decide whether $\left(S_{P}, A_{P}\right)$ can be obtained from $\left(S_{T}, A_{T}\right)$ by deleting

$k_{b}$ bases (together with their incident arcs) and $k_{a}$ arcs in addition, i.e.

whether there is a $k_{a}$-alignment $\varphi$ for $\left(S_{P}, A_{P} ; S_{T}, A_{T}\right)$.

Our aim is to prove the main result of the paper stated by Theorem 1 .

Theorem 1. There is an algorithm that solves any instance $\left(S_{P}, A_{P} ; S_{T}, A_{T} ; k_{a}\right)$ of the Almost Arc-Preserving SubSEQUence problem and runs in time $k_{b}^{O\left(k_{b}^{3}+k_{b} k_{a}\right)}\left|\left(S_{T}, A_{T}\right)\right|$ where $k_{b}=\left|S_{T}\right|-\left|S_{P}\right|$.

\subsection{Outline of the algorithm}

To prove Theorem 1, we present an algorithm that uses a bounded search tree technique in order to construct a $k_{a}$-alignment step by step. In certain situations, the algorithm might branch on a bounded number of possibilities to proceed with. Since both the number of such branchings and the possible directions of a branching will be bounded in terms of $k_{a}$ and $k_{b}$, the size of the resulting search tree will be bounded by a function of $k_{a}$ and $k_{b}$.

Actually, the algorithm described here has the following behavior: given an instance of APS, consisting of the arc-annotated sequences $\left(S_{P}, A_{P}\right)$ and $\left(S_{T}, A_{T}\right)$, and an integer $k_{a}$, it tries to construct a $k_{a}$-alignment $\varphi$ for $\left(S_{P}, A_{P} ; S_{T}, A_{T}\right)$. To do so, it fixes such a hypothetical solution $\varphi$, and looks for bases in $S^{\text {del }}(\varphi)$ and $\operatorname{arcs}$ in $A^{\mathrm{del}}(\varphi)$, which we will call removable bases and removable arcs of $\varphi$, resp. More precisely, our algorithm does one of the followings in linear time:

- it produces an arc-preserving alignment $\psi$ for $\left(S_{P}, A_{P} ; S_{T}, A_{T}\right)$ (note that $\psi$ is a $k_{a}$-alignment for $\left(S_{P}, A_{P} ; S_{T}, A_{T}\right)$ as well),

- it correctly rejects the instance, or

- it produces a removable base or a removable arc of $\varphi$.

In the last case, we can delete the given base or arc, and apply the algorithm to the obtained instance. Notice that one of the parameters $k_{a}$ and $k_{b}=\left|S_{T}\right|-\left|S_{P}\right|$ is decreased in the new instance. The presented algorithm will be shown to run in $f\left(k_{a}, k_{b}\right)\left|\left(S_{T}, A_{T}\right)\right|$ time for some functions $f$, which therefore implies Theorem 1 by proving that AAPS can be solved in $\left(k_{a}+k_{b}\right) f\left(k_{a}, k_{b}\right)\left|\left(S_{T}, A_{T}\right)\right|$ time.

Our algorithm might branch several times before producing an output as described above. Each such branch will be caused by guessing the answer to a question of the following form: given some position $p$ in $S_{P}$, what is the value of the position $\varphi(p) ?^{3}$ We interpret these branchings in the usual framework of bounded search trees: a branching happens when we do not know the exact value of a certain variable (such as the value of $\varphi(p)$ in the above example),

\footnotetext{
${ }^{3}$ In a few cases we will also need some additional branchings, described later on.
} 
and thus we have to investigate every possible value. A certain branch examines one possible value of the variable, and it produces a correct output if the given variable indeed has the value associated with this branch. Since the examined cases always cover every possibilities, this implies that the output will be correct in at least one of the branches.

Although our algorithm seems to be a straightforward application of the bounded search tree methodology used frequently in parameterized algorithms, we had to overcome many difficulties to avoid any possibility of using an unbounded number of such guesses. The presented algorithm will apply considerably sophisticated methods to keep the search tree bounded.

\subsection{Fragmentations and related concepts.}

Fragmentation. To describe our knowledge of the partially constructed $k_{a^{-}}$ alignment we have, we introduce a data structure called fragmentation. By iteratively refining the fragmentation, we can get closer and closer to actually determine a $k_{a}$-alignment. We write $\left|S_{P}\right|=n_{P}$ and $\left|S_{T}\right|=n_{T}$.

Recall that $\varphi$ is a fixed $k_{a}$-alignment for $\left(S_{P}, A_{P} ; S_{T}, A_{T}\right)$. For some $1 \leq i_{1} \leq$ $i_{2} \leq n_{P}$, we define the block $\left[i_{1}, i_{2}\right]$ in $S_{P}$ to be the set of positions $i_{1}, i_{1}+1, \ldots, i_{2}$, and we define blocks in $S_{T}$ similarly. Given a set of $f$ disjoint blocks $\left\{\left[p_{1}^{h}, p_{2}^{h}\right] \mid\right.$ $h \in[f]\}$ in $S_{P}$ and a set of $f$ disjoint blocks $\left\{\left[t_{1}^{h}, t_{2}^{h}\right] \mid h \in[f]\right\}$ in $S_{T}$, we let $F_{h}=\left(\left[p_{1}^{h}, p_{2}^{h}\right],\left[t_{1}^{h}, t_{2}^{h}\right]\right)$. We say that $\left\{F_{h} \mid h \in[f]\right\}$ is a fragmentation for $\varphi$, if

$-t_{1}^{h} \leq \varphi\left(p_{1}^{h}\right)$ and $\varphi\left(p_{2}^{h}\right) \leq t_{2}^{h}$ for each $h \in[f]$, and

$-p_{1}^{h+1}=p_{2}^{h}+1$ and $t_{1}^{h+1}=t_{2}^{h}+1$ for each $h \in[f-1]$.

We will call the element $F_{h}$ for some $h \in[f]$ a fragment. We define $\sigma\left(F_{h}\right)=$ $\left(t_{2}^{h}-t_{1}^{h}\right)-\left(p_{2}^{h}-p_{1}^{h}\right)$ and $\delta\left(F_{h}\right)=t_{1}^{h}-p_{1}^{h}$, which are both clearly non-negative integers. Note that $\delta\left(F_{h+1}\right)=\delta\left(F_{h}\right)+\sigma\left(F_{h}\right)$ holds for each $h \in[f-1]$. We say that a position $i \in\left[n_{P}\right]$ of $S_{P}$ is contained in the fragment $F_{h}$, if $p_{1}^{h} \leq i \leq p_{2}^{h}$.

We will say that a fragment $F$ is trivial if $\sigma(F)$ is zero, and non-trivial otherwise. We also call a position of $S_{P}$ trivial (or non-trivial) in a fragmentation, if the fragment containing it is trivial (or non-trivial, resp). Given fragmentation for $\varphi$ and a position $i$ in $S_{P}$, we will use the notation $i_{\text {left }}=i+\delta(F)$ and $i_{\text {right }}=i+\delta(F)+\sigma(F)$, where $F$ is the fragment containing $i$. Observe that

$$
i_{\text {left }} \leq \varphi(i) \leq i_{\text {right }}
$$

always holds. We will classify a position $i$ of $S_{P}$ as follows:

- If $\varphi(i)=i_{\text {left }}$, then $i$ is left-aligned.

- If $\varphi(i)=i_{\text {right }}$, then $i$ is right-aligned.

- If $\varphi(i)=j$ such that $i_{\text {left }}<j<i_{\text {right }}$, then $i$ is skew.

If $i$ is trivial, then only $\varphi(i)=i_{\text {left }}=i_{\text {right }}$ is possible. Thus, each trivial position must be both left- and right-aligned.

Notice that each fragment $F$ must contain exactly $\sigma(F)$ positions that are contained in $S^{\mathrm{del}}(\varphi)$. This implies the following bounds. 
Proposition 2. If $\mathcal{F}$ is a fragmentation for $\varphi$, then $\sum_{F \in \mathcal{F}} \sigma(F)=k_{b}$. In particular, $\mathcal{F}$ can have at most $k_{b}$ non-trivial fragments.

A marked fragmentation for $\varphi$ is a pair $(\mathcal{F}, M)$ formed by a fragmentation $\mathcal{F}$ for $\varphi$ and a set $M$ of positions in $S_{P}$ such that each $m \in M$ is a trivial position in $\mathcal{F}$. We say that the trivial positions contained in $M$ are marked.

For a fragment $F=\left(\left[p_{1}, p_{2}\right],\left[t_{1}, t_{2}\right]\right)$ we let $F^{\text {rev }}=\left(\left[p_{2}^{\text {rev }}, p_{1}^{\text {rev }}\right],\left[t_{2}^{\text {rev }}, t_{1}^{\text {rev }}\right]\right)$, hence a fragmentation $\mathcal{F}$ for $\varphi$ clearly yields a fragmentation $\mathcal{F}^{\text {rev }}=\left\{F^{\text {rev }} \mid F \in\right.$ $\mathcal{F}\}$ for $\varphi^{\text {rev }}$ as well. Note that if a position $i$ of $S_{P}$ is left-aligned (right-aligned) in $\mathcal{F}$, then the position $i^{\text {rev }}$ is right-aligned (left-aligned, resp.) in $\mathcal{F}^{\text {rev }}$.

Pairing arcs. Given a position $i$ in $S_{P}$, let us order the $\operatorname{arcs} c$ in $A_{P}^{+}(i)$ increasingly according to their right endpoint $c^{\text {end }}$. Similarly, we order the arcs in $A_{P}^{-}(i)$ increasingly according their left endpoint. In both cases, we break ties arbitrarily. Also, we order the $\operatorname{arcs}$ in $A_{T}^{+}(j)$ and $A_{T}^{-}(j)$ in the same way for each position $j$ in $S_{T}$. Now, we "pair" arcs in $A_{P}^{+}(i)$ with $\operatorname{arcs}$ in $A_{T}^{+}\left(i_{\text {left }}\right)$, and also $\operatorname{arcs}$ in $A_{P}^{-}(i)$ with $\operatorname{arcs}$ in $A_{T}^{-}\left(i_{\text {left }}\right)$ according to their ranking in this ordering. To this end, we construct the sets $R_{\text {left }}^{+}(i) \subseteq A_{P}^{+}(i) \times A_{T}^{+}\left(i_{\text {left }}\right)$ and $R_{\text {left }}^{-}(i) \subseteq A_{P}^{-}(i) \times A_{T}^{-}\left(i_{\text {left }}\right)$ in the following way. We put a pair $(c, d)$ into $R_{\text {left }}^{+}(i)$, if $c \in A_{P}^{+}(i), d \in A_{T}^{+}\left(i_{\text {left }}\right)$, and $c$ has the same rank (according to the above ordering) in $A_{P}^{+}(i)$ as the rank of $d$ in $A_{T}^{+}\left(i_{\text {left }}\right)$. Similarly, we put a pair $(c, d)$ into $R_{\text {left }}^{-}(i)$, if $c \in A_{P}^{-}(i), d \in A_{T}^{-}\left(i_{\text {left }}\right)$, and $c$ has the same rank in $A_{P}^{-}(i)$ as the rank of $d$ in $A_{T}^{-}\left(i_{\text {left }}\right)$. In addition, we define the sets $R_{\text {right }}^{+}(i)$ and $R_{\text {right }}^{-}(i)$ analogously, by substituting $i_{\text {right }}$ for $i_{\text {left }}$ in the above definitions. The key properties of these sets are summarized below.

Lemma 3. We know $\varphi\left(c^{\text {end }}\right)=d^{\text {end }}$ and $\varphi\left(c^{\text {start }}\right)=d^{\text {start }}$ in the following cases:

(1) If $(c, d) \in R_{\text {left }}^{+}(i)$ and $\left|A_{P}^{+}(i)\right|=\left|A_{T}^{+}\left(i_{\text {left }}\right)\right|$ for some left-aligned $i$.

(2) If $(c, d) \in R_{\text {left }}^{-}(i)$ and $\left|A_{P}^{-}(i)\right|=\left|A_{T}^{-}\left(i_{\text {left }}\right)\right|$ for some left-aligned $i$.

(3) If $(c, d) \in R_{\text {right }}^{+}(i)$ and $\left|A_{P}^{+}(i)\right|=\left|A_{T}^{+}\left(i_{\text {right }}\right)\right|$ for some right-aligned $i$.

(4) If $(c, d) \in R_{\text {right }}^{-}(i)$ and $\left|A_{P}^{-}(i)\right|=\left|A_{T}^{-}\left(i_{\text {right }}\right)\right|$ for some right-aligned $i$.

Arcs connecting two non-trivial fragments. Given two non-trivial fragments $F$ and $H$ of a fragmentation with $F$ preceding $H$, we define three disjoint subsets of those arcs of $A_{P}$ that start in a position of $F$ and end in a position of $H$. These sets will be denoted by $\mathcal{L}(F, H), \mathcal{R}(F, H)$, and $\mathcal{X}(F, H)$, and we construct them as follows. Suppose that $c=(f, h) \in A_{P}$ for some $f$ and $h$ contained in $F$ and $H$, respectively. We put $c$ in exactly one of these three sets, if $(c, d) \in R_{\text {left }}^{-}(h)$ for some arc $d \in A_{T}$ such that $f_{\text {left }} \leq d^{\text {start }} \leq f_{\text {right }}$. If $d^{\text {start }}=f_{\text {left }}$ then we put $c$ into $\mathcal{L}(F, H)$, if $d^{\text {start }}=f_{\text {right }}$ then we put $c$ into $\mathcal{R}(F, H)$, and if $f_{\text {left }}<d^{\text {start }}<f_{\text {right }}$ then we put $c$ into $\mathcal{X}(F, H)$.

By Lemma 3 , if the positions in $H$ are left-aligned, then the left endpoints of the $\operatorname{arcs}$ in $\mathcal{R}(F, H)$ must be right-aligned. Similarly, the left endpoints of the arcs in $\mathcal{X}(F, H)$ must be skew in such a case. Proposition 4 states these observations in a precise manner. Since we would like to ensure each position to be left-aligned, we will try to get rid of the $\operatorname{arcs}$ in $\mathcal{R}(F, H)$ and $\mathcal{X}(F, H)$. 
Proposition 4. Let $i$ be left-aligned, $\left|A_{P}^{-}(i)\right|=\left|A_{T}^{-}\left(i_{\text {left }}\right)\right|$, and $c \in A_{P}^{-}(i)$.

(1) If $c \in \mathcal{L}(F, H)$, then $c^{\text {start }}$ is left-aligned.

(2) If $c \in \mathcal{R}(F, H)$, then $c^{\text {start }}$ is right-aligned.

(3) If $c \in \mathcal{X}(F, H)$, then $c^{\text {start }}$ is skew.

We say that two positions $f_{1}, f_{2} \in\left[n_{P}\right]$ are conflicting for $(F, H)$, if $f_{1} \leq f_{2}$, $A_{P}^{+}\left(f_{1}\right) \cap \mathcal{R}(F, H) \neq \emptyset$ and $A_{P}^{+}\left(f_{2}\right) \cap \mathcal{L}(F, H) \neq \emptyset$. In such a case, we say that any $h \geq \max \left\{h_{1}, h_{2}\right\}$ in $H$ is conflict-inducing for $(F, H)$ (and for the conflicting pair $\left.\left(f_{1}, f_{2}\right)\right)$, where $h_{1}$ denotes the minimal position for which $\left(f_{1}, h_{1}\right) \in \mathcal{R}(F, H)$, and $h_{2}$ denotes the minimal position for which $\left(f_{2}, h_{2}\right) \in \mathcal{L}(F, H)$. Notice that if such a conflict-inducing $h$ is left-aligned, then both $h_{1}$ and $h_{2}$ are left-aligned. By Proposition 4, this implies that $f_{1}$ is right-aligned and $f_{2}$ is left-aligned. But since $f_{1}$ precedes $f_{2}$, this cannot happen. This implies the following observation.

Proposition 5. If a position $h$ is conflict-inducing for $(F, H)$ in a given fragmentation, then $h$ cannot be left-aligned.

In addition, if $\mathcal{L}(F, H) \neq \emptyset$, then let $L^{\max }(F, H)$ denote the largest position $f$ in $F$ for which $A_{P}^{+}(f) \cap \mathcal{L}(F, H) \neq \emptyset$. Let the L-critical position for $(F, H)$ be the smallest position $h$ contained in $H$ for which $\left(L^{\max }(F, H), h\right) \in \mathcal{L}(F, H)$. Similarly, if $\mathcal{R}(F, H) \neq \emptyset$, then let $R^{\min }(F, H)$ denote the smallest position $f$ in $F$ for which $A_{P}^{+}(f) \cap \mathcal{R}(F, H) \neq \emptyset$. Also, let the $R$-critical position for $(F, H)$ be the smallest position $h$ in $H$ for which $\left(R^{\min }(F, H), h\right) \in \mathcal{R}(F, H)$.

Now, a position $h$ in $H$ is LR-critical for $(F, H)$, if either $h$ is the R-critical position for $(F, H)$ and $\mathcal{L}(F, H)=\emptyset$, or $h=\max \left\{h_{L}, h_{R}\right\}$ where $h_{L}$ is the Lcritical and $h_{R}$ is the R-critical position for $(F, H)$. Note that both cases require $\mathcal{R}(F, H) \neq \emptyset$. Moreover, $H$ contains an LR-critical position for $(F, H)$, if and only if $\mathcal{R}(F, H) \neq \emptyset$. Intuitively, if an LR-critical position in $H$ is left-aligned, then this implies that some position in $F$ is right-aligned.

Note that the definitions of the sets $\mathcal{L}(F, H), \mathcal{R}(F, H)$, and $\mathcal{X}(F, H)$ together with the definitions connected to them as described above depend on the given fragmentation, so whenever the fragmentation changes, these must be adjusted appropriately as well. (In particular, arcs in $\mathcal{L}(F, H), \mathcal{R}(F, H)$, and $\mathcal{X}(F, H)$ must start and end in two different non-trivial fragments.)

Properties 1-9. Let $(\mathcal{F}, M)$ be a marked fragmentation for $\varphi$. Our aim is to ensure that the properties given below hold for each position in $S_{P}$. Intuitively, these properties mirror the expectation that every position should be left-aligned. Note that although we cannot decide whether $(\mathcal{F}, M)$ is a correct marked fragmentation without knowing the $k_{a}$-alignment $\varphi$, we are able to check whether these properties hold for some position $i$ in $(\mathcal{F}, M)$.

Property 1: $S_{P}[i]=S_{T}\left[i_{\text {left }}\right]$.

Property 2: If $i$ is non-trivial, then $\left|A_{P}^{+}(i)\right|=\left|A_{T}^{+}\left(i_{\text {left }}\right)\right|$ and $\left|A_{P}^{-}(i)\right|=\left|A_{T}^{-}\left(i_{\text {left }}\right)\right|$.

Property 3: If $i$ is non-trivial, then $A_{P}(y, i)=A_{T}\left(y_{\text {left }}, i_{\text {left }}\right)$ for any $y<i$ contained in the same fragment as $i$.

Property 4: If $i$ is non-trivial, then for every $(c, d) \in R_{\text {left }}^{+}(i)$ such that $c^{\text {end }}=y$ is non-trivial, $y_{\text {left }} \leq d^{\text {end }} \leq y_{\text {right }}$ holds. Also, for every $(c, d) \in R_{\text {left }}^{-}(i)$ such that $c^{\text {start }}=y$ is non-trivial, $y_{\text {left }} \leq d^{\text {start }} \leq y_{\text {right }}$ holds. 
Property 5: No arc in $\mathcal{X}(F, H)$ for some $(F, H)$ ends at $i$.

Property 6: $i$ is not conflict-inducing for any $(F, H)$.

Property 7: $i$ is not LR-critical for any $(F, H)$.

Property 8: If $i$ is non-trivial, then for every $(c, d) \in R_{\text {left }}^{+}(i)$ such that $c^{\text {end }}$ $=y$ is non-trivial, $d^{\text {end }}=y_{\text {left }}$ holds. Also, for every $(c, d) \in R_{\text {left }}^{-}(i)$ such that $c^{\text {start }}=y$ is non-trivial, $d^{\text {start }}=y_{\text {left }}$ holds.

Property 9: If $i$ is non-trivial, then for each marked position $m \in M, A_{P}(i, m)=$ $A_{T}\left(i_{\text {left }}, m_{\text {left }}\right)$ holds if $m>i$, and $A_{P}(m, i)=A_{T}\left(m_{\text {left }}, i_{\text {left }}\right)$ holds if $m<i$.

Observe that each of these properties depend on the fragmentation $\mathcal{F}$, and Property 9 depends on the set of marked positions $M$ as well. Also, if some property holds for a position $i$ in $(\mathcal{F}, M)$, then this does not imply that the property holds for $i^{\text {rev }}$ in $\left(\mathcal{F}^{\text {rev }}, M^{\text {rev }}\right)$, as most of these properties are not symmetric. For example, $i_{\text {left }}$ and $i_{\text {right }}$ both have a different meaning in the fragmentation $\mathcal{F}$ and in $\mathcal{F}^{\text {rev }}$. We say that a position $i \in\left[n_{P}\right]$ violates Property $\ell(1 \leq \ell \leq 9)$ in a marked fragmentation $(\mathcal{F}, M)$, if Property $\ell$ does not hold for $i$ in $(\mathcal{F}, M)$.

If the first eight properties hold for each position both in $(\mathcal{F}, M)$ and in $\left(\mathcal{F}^{\text {rev }}, M^{\text {rev }}\right)$, then we say that $(\mathcal{F}, M)$ is 8-proper. We say that $(\mathcal{F}, M)$ is proper, if it is 8-proper and Property 9 holds hold for each position of $S_{P}$ in $(\mathcal{F}, M)$. Note that we do not care whether Property 9 holds for the positions in the reversed instance, so $(\mathcal{F}, M)$ is proper even if Property 9 does not hold in $\left(\mathcal{F}^{\text {rev }}, M^{\text {rev }}\right)$.

\subsection{Description of the algorithm}

We start with a marked fragmentation where $M=\emptyset$ and the fragmentation contains only the unique fragment $\left(\left[1, n_{P}\right],\left[1, n_{T}\right]\right)$, which is non-trivial if $k_{b}>0$. Given a marked fragmentation $(\mathcal{F}, M)$, we do the following: if one of Properties $1,2, \ldots, 9$ does not hold for some position $i$ in $(\mathcal{F}, M)$ or one of the first eight properties does not hold for some $i$ in the reversed marked fragmentation $\left(\mathcal{F}^{\mathrm{rev}}, M^{\mathrm{rev}}\right)$, then we will either reject the instance, output a removable base of $\varphi$, or modify the given marked fragmentation. If the given marked fragmentation is proper, the algorithm returns an output using Lemmas 9 and 10.

To do this, in each step we choose the first property violated by a position either in $(\mathcal{F}, M)$ or in $\left(\mathcal{F}^{\text {rev }}, M^{\text {rev }}\right)$. Observe that we can assume w.l.o.g. that there is an $\ell(1 \leq \ell \leq 9)$ such that Properties $1, \ldots, \ell-1$ hold for each position both in $(\mathcal{F}, M)$ and in $\left(\mathcal{F}^{\text {rev }}, M^{\text {rev }}\right)$, but Property $\ell$ is violated by a position in $S_{P}$ in $(\mathcal{F}, M)$, otherwise we simply reverse the instance. (We only reverse it if this condition is not true.)

Given $\ell$, the algorithm takes the first position $i$ violating Property $\ell$, and branches on choosing $\varphi(i)$ according to $i_{\text {left }} \leq \varphi(i) \leq i_{\text {right }}$. By Proposition 2, this results in at most $k_{b}+1$ directions. Next, the algorithm handles each of the cases in a different manner, according to whether $i$ turns out to be leftaligned, right-aligned, or skew. We consider these cases in a general way that is essentially independent from $\ell$, and mainly relies on the type of $i$. We suppose that $i$ is contained in a fragment $F^{i}=\left(\left[p_{1}, p_{2}\right],\left[t_{1}, t_{2}\right]\right)$. 
Extremal cases. Assume that $i=p_{1}$ and $i$ is skew or right-aligned, or $i=p_{2}$ and $i$ is skew or left-aligned. In these cases, we can find at least one removable base of $\varphi$. First, if $i=p_{1}$ and $i$ is skew or right-aligned, then each base $S_{T}[j]$ must be deleted for each $j$ where $t_{1} \leq j<\varphi(i)$. Second, if $i=p_{2}$ and $i$ is skew or left-aligned, then $S_{T}[j]$ must be deleted for each $j$ where $\varphi(i)<j \leq t_{2}$.

Skew position. Suppose that $i>p_{1}$ and $j$ is skew, meaning that $\varphi(i)=j$ for some $j$ with $i_{\text {left }}<j<i_{\text {right }}$. In this case, we can divide the fragment $F^{i}$, or more precisely, we can delete $F^{i}$ from the fragmentation $\mathcal{F}$ and add the new fragments $\left(\left[p_{1}, i-1\right],\left[t_{1}, j-1\right]\right)$ and $\left(\left[i, p_{2}\right],\left[j, t_{2}\right]\right)$. Note that the newly introduced fragments are non-trivial by the bounds on $j$. We also modify $M$ by declaring every trivial position of the fragmentation to be marked (no matter whether it was marked or not before). Observe that the number of non-trivial fragments increases in this step. By Proposition 2, this can happen at most $k_{b}-1$ times.

Left-aligned position. Lemma 6 summarizes our results that show how to deal with the case when $i$ is left-aligned and $i<p_{2}$. The proof of this lemma is essential in the correctness of our algorithm.

Lemma 6. Suppose that Property $\ell(1 \leq \ell \leq 9)$ does not hold for some $i \in\left[n_{P}\right]$ in the marked fragmentation $(\mathcal{F}, M)$, but all the previous properties hold for each position both in $(\mathcal{F}, M)$ and in $\left(\mathcal{F}^{\mathrm{rev}}, M^{\mathrm{rev}}\right)$. If $i$ is left-aligned, then depending on $\ell$, we can do one of the followings in linear time (without any branchings):

A) reject correctly,

B) output a removable arc of $\varphi$,

$C$ ) find that $i$ is incident to a removable arc of $\varphi$ (this only happens if $\ell=2$ ),

D) produce a skew position $i^{\prime}$, or

E) produce a set $N$ of at most $2 k_{b}-1$ positions in $S_{T}$ such that $N \cap S^{\operatorname{del}}(\varphi) \neq \emptyset$.

In Case $\mathrm{A}$ or $\mathrm{B}$, we reject or output a removable $\operatorname{arc}$ of $\varphi$.

In Case $\mathrm{C}$, we put the non-trivial position $i$ in a set $W$, which will only store positions in $S_{T}$ that are incident to a removable arc of $\varphi$. (We set $W=\emptyset$ initially.) Whenever Case C happens, we examine whether $|W| \leq 2 k_{a}$. If not, then we reject the input. This is correct, since there can be at most $k_{a}$ removable arcs of $\varphi$, and each such arc is incident to two bases.

If $|W| \leq 2 k_{a}$ holds, then we modify the given fragmentation, replacing $F^{i}$ by new fragments $F_{1}=\left(\left[p_{1}, i\right],\left[t_{1}, i_{\text {left }}\right]\right)$ and $F_{2}=\left(\left[i+1, p_{2}\right],\left[i_{\text {left }}+1, t_{2}\right]\right)$. By $\varphi(i)=i_{\text {left }}$, this yields a fragmentation for $\varphi$. Note that $F_{1}$ is trivial and $F_{2}$ is non-trivial. We mark each position of $F_{2}$, putting them into $M$. We refer to this operation as a left split at $i$. Since $i$ becomes trivial in $F_{1}$, each position can be placed into $W$ at most once. Thus, Case $\mathrm{C}$ can happen at most $2 k_{a}$ times without rejecting.

In Cases D and E, we might branch into a bounded number of additional branches. In Case D, we branch on those choices of $\varphi\left(i^{\prime}\right)$ where $i^{\prime}$ is indeed skew, which means $\sigma\left(F^{i}\right)-1 \leq k_{b}-1$ directions, and we handle each branch according to the way described above (dividing one fragment at the skew position $i^{\prime}$ ). In Case $\mathrm{E}$, we branch into at most $2 k_{b}-1$ directions on choosing a removable base of $\varphi$ from $N$ and outputting it. 
Note that Case $\mathrm{D}$ or $\mathrm{E}$ can happen at most $k_{b}$ times, by our observation that a skew position can only be found at most $k_{b}-1$ times.

We remark that if $i$ is trivial, then we treat it as left-aligned.

Right-aligned position. Suppose that $i>p_{1}$ and $i$ is right-aligned. In this case, we replace $F^{i}$ by new fragments $F_{1}=\left(\left[p_{1}, i-1\right],\left[t_{1}, i_{\text {right }}-1\right]\right)$ and $F_{2}=\left(\left[i, p_{2}\right],\left[i_{\text {right }}, t_{2}\right]\right)$. This yields a fragmentation where $F_{1}$ is non-trivial and $F_{2}$ is trivial. We refer to this operation as performing a right split at $j$. If this happens because $i$ violated Property $\ell$ for some $\ell \leq 8$, then we mark every trivial position (including those contained in $F_{2}$ ), by putting them into $M$. If $\ell=9$, then we do not modify $M$, so the trivial positions of $F_{2}$ will not be marked.

The above process either produces a removable base of $\varphi$, rejects correctly, or ends by providing a marked fragmentation that is proper. In the remaining steps of the algorithm, the set $M$ will never be modified, and the only possible modification of the actual fragmentation will be to perform a right split.

Given a proper marked fragmentation $(\mathcal{F}, M)$, we make use of Lemma 9 below. This lemma gives sufficient conditions to do one of the followings.

- Find out that some non-trivial position $i$ is right-aligned. In this case, we perform a right split at $i$ in the actual fragmentation.

- Find a removable arc of $\varphi$.

- Reject correctly.

Our algorithm applies Lemma 9 repeatedly, until it either stops (by rejecting or outputting a removable arc of $\varphi$ ), or finds that none of the conditions of Lemma 9 apply. Before stating this lemma, we need two more important observations. First, Lemma 7 shows that the repeated application of Lemma 9 results in a proper fragmentation. Second, Lemma 8 states some useful invariants that hold for each fragmentation obtained by us after a proper fragmentation is achieved.

Lemma 7. If $(\mathcal{F}, M)$ is proper and $\mathcal{F}^{\prime}$ is obtained by applying an arbitrary number of right splits to $\mathcal{F}$, then $\left(\mathcal{F}^{\prime}, M\right)$ is proper as well.

Lemma 8. Let $(\mathcal{F}, M)$ be a 8-proper marked fragmentation whose trivial positions are all marked. Suppose that $\mathcal{F}^{\prime}$ is obtained by applying an arbitrary number of right splits to the fragmentation $\mathcal{F}$.

(1) For each $i$ that is not marked $\left(i \in\left[n_{P}\right] \backslash M\right)$, both $A_{P}^{+}(i)=A_{T}^{+}\left(i_{\text {right }}\right)$ and $A_{P}^{-}(i)=A_{T}^{-}\left(i_{\text {right }}\right)$ hold in $\left(\mathcal{F}^{\prime}, M\right)$.

(2) Suppose that neither $i$ nor $j$ is marked $\left(i, j \in\left[n_{P}\right] \backslash M\right)$ and $c=(i, j) \in A_{P}$. If $(c, d) \in R_{\text {right }}^{+}(i)$ for some $d \in A_{T}^{+}\left(i_{\text {right }}\right)$, then $d^{\text {end }}=j_{\text {right }}$. Similarly, if $(c, d) \in R_{\text {right }}^{-}(j)$ for some $d \in A_{T}^{-}\left(j_{\text {right }}\right)$, then $d^{\text {start }}=i_{\text {right }}$.

Now, we can state Lemma 9.

Lemma 9. Let $(\mathcal{F}, M)$ be a proper marked fragmentation for $\varphi$ obtained by our algorithm, and let $a, b \in\left[n_{P}\right]$.

(i) Suppose that $a$ is trivial but not marked and $b$ is non-trivial. If $(a, b) \in A_{P}$ 
or $(b, a) \in A_{P}$, then $b$ is right-aligned.

(ii) If $a$ and $b$ are trivial, $a<b$ and $A_{P}(a, b) \neq A_{T}\left(a_{\text {left }}, b_{\text {left }}\right)$, then we can either reject or output a removable arc of $\varphi$.

After applying Lemma 9 repeatedly, the algorithm either stops by rejecting or outputting a removable arc of $\varphi$, or it finds that neither of the conditions (i) and (ii) of Lemma 9 holds. Let $(\mathcal{F}, M)$ be the final marked fragmentation obtained. Note that the algorithm does not modify the set $M$ of marked trivial positions when applying Lemma 9, and it can only modify the actual fragmentation by performing a right split. Hence, Lemma 7 yields that $(\mathcal{F}, M)$ is proper.

Using $(\mathcal{F}, M)$, Lemma 10 claims that we can find an arc-preserving alignment for $\left(S_{P}, A_{P} ; S_{T}, A_{T}\right)$ in linear time. Hence, the final step of our algorithm, finishing its description, is to output this arc-preserving alignment.

Lemma 10. Let $(\mathcal{F}, M)$ be a proper marked fragmentation for $\varphi$ obtained by the algorithm. If none of the conditions of Lemma 9 holds, then we can produce an arc-preserving alignment $\psi$ for $\left(S_{P}, A_{P} ; S_{T}, A_{T}\right)$ in linear time.

Proof. We show that defining $\psi(i)=i_{\text {left }}$ for each position $i \in\left[n_{P}\right]$ fulfills the requirements. For this, we have to prove $S_{P}[i]=S_{T}\left[i_{\text {left }}\right]$ for each position $i \in\left[n_{P}\right]$, and $A_{P}(i, j)=A_{T}\left(i_{\text {left }}, j_{\text {left }}\right)$ for each two positions $i \neq j \in\left[n_{P}\right]$.

First, as Property 1 holds for each position in $\mathcal{F}$, we know $S_{P}[i]=S_{T}\left[i_{\text {left }}\right]$ for each $i \in\left[n_{P}\right]$. It remains to show $A_{P}(i, j)=A_{T}\left(i_{\text {left }}, j_{\text {left }}\right)$ for each $i \neq j \in\left[n_{P}\right]$. If both $i$ and $j$ are trivial positions, then this is true because the conditions of (ii) in Lemma 9 do not apply. If both $i$ and $j$ are non-trivial, then $A_{P}(i, j)=$ $A_{T}\left(i_{\text {left }}, j_{\text {left }}\right)$ again holds, by Properties 2 and 8 for $j$. Now, if $i$ is non-trivial but $j$ is trivial and marked (or vice versa), then Property 9 implies the required equality. Finally, if one of $i$ and $j$ is non-trivial and the other one is trivial but not marked, then $A_{P}(i, j)=0$ holds, since (i) of Lemma 9 is not applicable.

\subsection{Analysis of the algorithm}

In this section, we give some hints how to analyse the running time of the presented algorithm. The following lemma, stating the key properties of the our algorithm, proves Theorem 1 .

Lemma 11. Let $\left(S_{P}, A_{P}, S_{T}, A_{T}, k_{a}\right)$ be the given instance of APS. The presented algorithm branches into at most $f\left(k_{a}, k_{b}\right)$ directions in total for some function $f$ such that in each branch it does one of the followings (supposing that the conditions of the given branch do hold):

- it gives an arc-preserving alignment $\psi$ of $\left(S_{P}, A_{P} ; S_{T}, A_{T}\right)$,

- it correctly rejects the instance, or

- it outputs a removable base or a removable arc of $\varphi$.

Moreover, each branch takes linear time in the size of the input. 
Although we do not prove Lemma 11 due to lack of space, we give the most important definitions used in the proof.

Given a fragmentation $\mathcal{F}$ for $\varphi$, a fragment $F \in \mathcal{F}$, and some $\ell(1 \leq \ell \leq 8)$, let $\pi(\mathcal{F}, F, \ell)$ be 1 if Property $\ell$ holds for each position $i$ in $F$, and 0 otherwise. Let $N(\mathcal{F})$ denote the set of non-trivial fragments in $\mathcal{F}$. We define the measure $\mu(\mathcal{F})$ of a given fragmentation $\mathcal{F}$ for $\varphi$ as follows:

$$
\mu(\mathcal{F})=\sum_{1 \leq \ell \leq 8}\left(\sum_{F \in N(\mathcal{F})} \pi(\mathcal{F}, F, \ell)+\sum_{F \in N\left(\mathcal{F}^{\mathrm{rev}}\right)} \pi\left(\mathcal{F}^{\mathrm{rev}}, F, \ell\right)\right) .
$$

Note that $\mu(\mathcal{F})=\mu\left(\mathcal{F}^{\text {rev }}\right)$ is trivial, so reversing a fragmentation does not change its measure. The importance of this definition is shown by Lemma 12.

Lemma 12. Let $\mathcal{F}_{1}, \ldots, \mathcal{F}_{t}, \mathcal{F}_{t+1}$ be a series a fragmentations such that for each $i \in[t]$ the algorithm obtains $\mathcal{F}_{i+1}$ from $\mathcal{F}_{i}$ by applying a left or a right split at a position $j_{i}$ violating Property $\ell_{i}$ in $\mathcal{F}_{i}$. Then (1) $\mu\left(\mathcal{F}_{i+1}\right) \geq \mu\left(\mathcal{F}_{i}\right)$ for each $i \in[t]$, and (2) if $\mu\left(\mathcal{F}_{1}\right)=\mu\left(\mathcal{F}_{t}\right)$, then $t \leq k_{b}$ holds.

\section{References}

1. J. Alber, J. Gramm, J. Guo, and R. Niedermeier. Computing the similarity of two sequences with nested arc annotations. Theor. Comput. Sci., 312(2-3):337-358, 2004.

2. G. Blin, G. Fertin, R. Rizzi, and S. Vialette. What makes the Arc-Preserving Subsequence problem hard? In IWBRA'05: Proceedings of the 5th Int. Workshop on Bioinformatics Research and Applications, volume 3515 of Lecture Notes in Computer Science, pages 860-868. Springer-Verlag, 2005.

3. P. Damaschke. A remark on the subsequence problem for arc-annotated sequences with pairwise nested arcs. Inf. Process. Lett., 100(2):64-68, 2006.

4. R. G. Downey and M. R. Fellows. Parameterized complexity. Monographs in Computer Science. Springer-Verlag, New York, 1999.

5. P. A. Evans. Algorithms and complexity for annotated sequence analysis. $\mathrm{PhD}$ thesis, University of Victoria, Canada, 1999.

6. P. A. Evans. Finding common subsequences with arcs and pseudoknots. In $C P M$ '99: Proceedings of the 10th Annual Symposium on Combinatorial Pattern Matching, volume 1645 of Lecture Notes in Computer Science, pages 270-280. SpringerVerlag, 1999.

7. J. Flum and M. Grohe. Parameterized Complexity Theory. Texts in Theoretical Computer Science. An EATCS Series. Springer-Verlag, New York, 2006.

8. J. Gramm, J. Guo, and R. Niedermeier. Pattern matching for arc-annotated sequences. ACM Trans. Algorithms, 2(1):44-65, 2006.

9. T. Jiang, G. Lin, B. Ma, and K. Zhang. The longest common subsequence problem for arc-annotated sequences. J. Discrete Algorithms, 2(2):257-270, 2004.

10. G. Lin, Z.-Z. Chen, T. Jiang, and J. Wen. The longest common subsequence problem for sequences with nested arc annotations. J. Comput. Syst. Sci., 65(3):465480, 2002.

11. B. Ma, L. Wang, and K. Zhang. Computing similarity between rna structures. Theor. Comput. Sci., 276(1-2):111-132, 2002.

12. D. Marx and I. Schlotter. Cleaning interval graphs. CoRR, abs/1003.1260, 2010. arXiv:1003.1260 [cs.DS]. 